\title{
Adrenomedullin improves intestinal epithelial barrier function by downregulating myosin light chain phosphorylation in ulcerative colitis rats
}

\author{
ZAIFENG YI, HENG FAN, XINGXING LIU, QING TANG, DONGMEI ZUO and JIA YANG \\ Department of Integrated Traditional Chinese and Western Medicine, Union Hospital, Tongji Medical College, \\ Huazhong University of Science and Technology, Wuhan, Hubei 430030, P.R. China
}

Received August 7, 2014; Accepted May 8, 2015

DOI: $10.3892 / \mathrm{mmr} .2015 .3887$

\begin{abstract}
Adrenomedullin (AM) is a pivotal endogenous vasoactive peptide, which can maintain epithelial barrier function in inflammatory bowel disease. Myosin light chain kinase (MLCK)-dependent phosphorylated myosin light chain kinase (p-MLC) is a key regulator of intestinal barrier function. The aim of the present study was to investigate the effect and mechanism of AM on the intestinal epithelial barrier in a rat model of ulcerative colitis (UC) induced by 2,4,6-trinitro-benzene-sulfonic acid (TNBS). A total of 21 male Sprague-Dawley rats were randomly divided into the following three groups and administered different agents for 7 days: The normal group (water and saline), model group (TNBS and saline) and the AM group (TNBS and AM; $1.0 \mu \mathrm{g})$. The weight of rats was recorded every day. Serum tumor necrosis factor- $\alpha$ (TNF- $\alpha$ ) and interleukin-6 (IL-6) levels were detected using ELISA kits. Colon tissue was collected for the assessment of histological alterations. The protein expression of MLCK, p-MLC and zonula occludens-1 (ZO-1) was examined by western blot analysis. Intestinal epithelial tight junctions were examined using transmission electron microscopy. The results demonstrated that in colitis model rats, the expression of TNF- $\alpha$, IL-6, MLCK and p-MLC significantly increased compared with normal rats. In addition, the expression of ZO-1 decreased $(\mathrm{P}<0.05)$ and intestinal epithelial cell permeability increased. Following AM administration, TNF- $\alpha$, IL-6, MLCK and p-MLC expression significantly decreased compared with the model rats, the expression of ZO-1 increased $(\mathrm{P}<0.05)$ and intestinal epithelial cell permeability reduced. These data indicate a protective
\end{abstract}

Correspondence to: Professor Heng Fan, Department of Integrated Traditional Chinese and Western Medicine, Union Hospital, Tongji Medical College, Huazhong University of Science and Technology, 1277 Jiefang Avenue, Wuhan, Hubei 430030, P.R. China

E-mail: fheng2003@163.com

Key words: adrenomedullin, ulcerative colitis, cytokine, myosin light chain kinase, myosin light chain effect of AM on intestinal epithelial barrier dysfunction via suppression of inflammatory cytokines and downregulation of MLCK-p-MLC in TNBS-induced UC. In conclusion, AM/MLCK-p-MLC may be an important signaling pathway in the occurrence and development of UC.

\section{Introduction}

Ulcerative colitis (UC) is an important type of inflammatory bowel disease (IBD), which is a chronic non-specific autoimmune disease. Although environmental factors, infections and immunological and psychological factors can lead to the occurrence of the disease, the specific pathogenesis remains to be elucidated.

Intestinal mucosal barrier damage has been recognized as one of the key factors in the pathogenesis of IBD. A single layer of epithelial cells covering the intestinal mucosal surface, form a physical barrier to withstand external stimuli and infections and maintain homeostasis between the intestinal cavity and internal tissues $(1,2)$. The function of intestinal mucosal barrier protection is often referred to as intestinal permeability in IBD (3). Hyperactivity and increased permeability of intestinal epithelium is an important characteristic of IBD (4). A previous study confirmed that colonic epithelial barrier dysfunction is considered to be important in the initiation of intestinal inflammation (5). The main functional characteristic of intestinal epithelial barrier dysfunction is intestinal permeability alterations, which not only easily lead to disease, but may also be an important reason for the onset of delayed healing $(6,7)$.

Barrier function is dynamic and can be adjusted by a variety of physiological and pathophysiological stimuli (8). Several lines of evidence support the theory that myosin light chain kinase (MLCK)-dependent phosphorylation of MLC has a central role in the pathogenesis of IBD (9-11). The levels of MLCK are positively associated with disease severity in UC. Increased MLCK expression can lead to the destruction of tight junctions (TJs), which is associated with increased intestinal permeability (12). This phenomenon can be prevented by MLCK inhibitors (13).

Adrenomedullin (AM) is an endogenous vasoactive peptide, which is closely associated with cell metabolism, 
immune function and endocrine and cardiovascular function, and is important in a number of tissues and organs (14). Previous studies have suggested that intestinal epithelial cells may be an important target for the protective effect of AM. Sakata et al found that AM had a higher expression in the cecum and colon and may have an important protective role in the colon (15), however, the specific mechanisms involved remain to be elucidated. Prior studies have demonstrated that AM may exert a protective effect in the gut by its anti-inflammatory properties and by suppressing the generation of bacteria (16-18). Other studies also suggest that AM can maintain epithelial barrier function via constraining inflammatory cytokine production and altering the balance of T cells (4,19-21). These data indicate that AM is an important protective peptide for intestinal epithelial cells and is important in the protection of the intestinal mucosal barrier. Based on the crucial role of MLCK in the intestinal mucosal barrier, the present study aimed to evaluate the effect of AM on intestinal permeability and on the MLCK-p-MLC pathway in a UC animal model induced by 2,4,6-trinitro-benzene-sulfonic acid (TNBS), and to understand its relative contribution to disease pathogenesis and further clarify the mechanism of AM in the disease process of UC.

\section{Materials and methods}

Ethics statement. Animal experiments were approved by the ethics committee of Tongji Medical College Affiliated to Huazhong University of Science and Technology (Wuhan, China) and conformed to the provisions of the Declaration of Helsinki (as revised in Edinburgh 2000). In addition, all animals received humane care and the procedures were performed in accordance with the Principles of Laboratory Animal Care.

Animals. A total of 21 male Sprague-Dawley rats weighing 200-250 g (Beijing HFK Bioscience Co., Ltd., Beijing, China) were used for the experiments. Rats were maintained at the Animal Experimental Center of Huazhong University of Science and Technology and kept in specific pathogen free air at a temperature of $22^{\circ} \mathrm{C}$ with a relative humidity of $50 \%$ and a $12 \mathrm{~h}$ light and dark cycle. Rats were randomly divided into three groups, namely, the normal, model and AM group, with seven rats in each group.

Introduction of colitis. Male Sprague-Dawley rats were induced with colitis as previously described (22). Briefly, rats were fasted for $24 \mathrm{~h}$, then TNBS (Sigma-Aldrich, St. Louis, MO, USA; $5 \%, 0.6 \mathrm{ml})$ was integrated in ethanol $(50 \%$, $0.25 \mathrm{ml}$ ) and delivered into the lumen of the colon via a $\sim 15 \mathrm{~cm}$ diameter $2.0 \mathrm{~mm}$ rubber infusion tube inserted into the rectum to a depth of $\sim 8 \mathrm{~cm}$ from the anal verge.

Administration of $A M$. The recombinant AM used in the present study was purchased from Sino Biological Inc. (Beijing, China). After $48 \mathrm{~h}$ of TNBS administration, AM (1.0 $\mu \mathrm{g}$ of AM diluted in $1.0 \mathrm{ml}$ of saline) was delivered into the lumen of the colon via a $15 \mathrm{~cm}$ long rubber infusion tube inserted into the rectum to a depth of $\sim 8 \mathrm{~cm}$ from the anal verge. Non AM-treated rats were administered $1.0 \mathrm{ml}$ of saline without AM. AM and saline were administered once a day for 7 days.

Histological analysis. Colon tissues were fixed in 4\% paraformaldehyde and histological alterations were observed by hematoxylin and eosin (H\&E) staining in routinely processed paraffin-embedded sections.

ELISA evaluation of TNF- $\alpha$ and IL-6. Blood samples were acquired by cardiac puncture and used for the evaluation of ELISA. ELISA for TNF- $\alpha$ (rat TNF- $\alpha$ ELISA kit, cat. no. ERC102a; NeoBioscience, Shenzhen, China) and IL-6 (rat IL-6 ELISA kit, cat. no. ERC003; NeoBioscience) was performed according to the manufacturer's instructions. The peroxidase reaction was terminated by the addition of $100 \mu \mathrm{l}$ $1 \mathrm{~N} \mathrm{H}_{2} \mathrm{SO}_{4}$. The absorbance was examined using a microplate reader (synergy 2; BioTek Instruments, Inc., Winooski, VT, USA) at $450 \mathrm{~nm}$.

Western blot analysis. Monoclonal rabbit anti-MLCK antibody (cat. no. ab76092; Abcam, Cambridge, MA, USA) and polyclonal rabbit anti-myosin light chain (phospho S20) antibody (cat. no. ab2480; Abcam) were used to assess the expression of MLCK and p-MLC. Monoclonal rat anti-ZO-1 antibody (cat. no. MABT11; Millipore, Billerica, MA, USA) was used to assess ZO-1 expression. Proteins were extracted from intestinal mucosa. Total protein $(50 \mu \mathrm{g})$ from each sample was detected by sodium dodecyl sulfate polyacrylamide gel electrophoresis, respectively. Subsequently, the gels were transferred onto polyvinylidene difluoride membranes (Millipore), which were later blocked in Tris-buffered saline Tween-20 containing 5\% fat-free dry milk for $2 \mathrm{~h}$ or overnight at room temperature. The blots were performed using primary antibodies against rat MLCK (diluted 1:1,000), rat p-MLCK (diluted 1:1,000) and rat ZO-1 (diluted 1:1,000), respectively, followed by incubation with horseradish peroxidase-linked goat anti-rabbit (diluted 1:10,000; cat. no. LK2001; Tianjin Sungene Biotech Co., Ltd., Tianjin, China) or rabbit anti-rat (1:50,000; cat. no. BA1058; Wuhan Boster Biological Technology, Ltd., Wuhan, China) secondary antibodies. Finally, the extended signals of protein-antibody complexes were detected through exposure to X-ray film. To standardize the protein expression, $\beta$-actin was conducted by immunoblotting techniques as an internal control, respectively. BandScan 5.0 software (Glyko Inc., Novato, CA, USA) was used to analyze the gray scale values for different bands.

Measurement of transmission electron microscopy (TEM). The integrity of the Tjs of epithelial cells was assessed by measuring TEM. The rats were sacrificed by intraperitoneal injection of $0.3 \mathrm{ml} / 100 \mathrm{~g} \mathrm{10 \%}$ chloral hydrate (Wuhan Union Hospital Pharmacy, Wuhan, China), and then $\sim 1 \mathrm{~cm}$ square colon tissue was obtained using a sharp blade. The colon tissue was fixed in $2.5 \%$ glutaraldehyde-buffered fixative (absolutely avoid extrusion or using scissors or forceps to uptake) and pre-fixed for 5-10 min. The bowel was then cut into an area of $1 \mathrm{x} 1 \mathrm{~mm}$ tissue, fixed in $2.5 \%$ glutaraldehyde-buffered fixative for 2-4 h, using $0.1 \mathrm{M}$ phosphate buffer ( $\mathrm{pH} 7.4$ ) and washed twice, for $20 \mathrm{~min}$ each. The tissue was then postfixed in $1 \%$ osmium tetroxide for $2 \mathrm{~h}$, dehydrated in acetone, and embedded in epoxy resin 618. 
Areas of interest were selected from semi-thin sections, which were initally stained with aqueous uranyl acetate followed by lead citrate. The sections were then detected under TEM (VEGA3 LMU; Tescan, Brno, Czekh Republic).

Statistical analysis. Experiments were duplicated at least three times and the values are presented as the mean \pm standard deviation. One-way analysis of variance was used to analyze the statistical differences. Statistical analysis was performed using SPSS 13.0 software (SPSS, Inc., Chicago, IL, USA). $\mathrm{P}<0.05$ was considered to indicate a statistically significant difference.

\section{Results}

Symptoms of rats in different groups. The activity, diet, stool traits, and body weight of each rat were observed. Rats administered TNBS exhibited bradykinesia, reduced food intake, sustained weight loss, and their stools contained blood and mucus, as compared with the normal rats. Conversely, the effects of TNBS were markedly ameliorated in the AM-treated rats, and the reduced body weight of the rats was also improved, as compared with the untreated group (Fig. 1).

Histological variations. Histological examination demonstrated a normal morphology in normal rats (Fig. 2A). However, apparent effusion and adhesion, a shorter bowel length and a thinner intestinal wall was observed in the model group, and a section of the colon revealed superficial ulcers, bleeding and erosion in the mucosa (Fig. 2B). By contrast, following AM administration pathological alterations were significantly less severe than in the model group and the superficial ulcers of intestinal mucosa were significantly reduced (Fig. 2C). H\&E staining demonstrated mucosal atrophy, partial microvilli loss, edema of the lamina propria and marked inflammatory cell infiltration in the TNBS-induced group (Fig. 3A) compared with the normal group, which exhibited a clear intestinal wall structure and regularly arranged microvilli (Fig. 3B). Edema and inflammatory cell infiltration were reduced following AM administration (Fig. 3C).

TNF- $\alpha$ and IL-6. ELISA was used to detect the expression of TNF- $\alpha$ and IL-6. The results demonstrated that the expression of TNF- $\alpha$ and IL- 6 in the serum of model rats was significantly higher than in the normal group $(\mathrm{P}<0.05)$, whereas a lower expression was observed following $\mathrm{AM}$ treatment compared with the model group ( $\mathrm{P}<0.05$; Fig. 4).

MLCK and p-MLC protein. MLC has a crucial role in barrier function, an increase in the phosphorylation of MLC can mediate epithelial barrier dysfunction. The present study therefore assessed the effect of AM on MLC phosphorylation using western blot analysis. As shown in Fig. 5, MLCK and p-MLC expression significantly increased in the model group compared with normal rats $\left({ }^{*} \mathrm{P}<0.05\right)$. However, following AM administration, MLCK and p-MLC expression significantly decreased compared with the untreated colitis rats $(\mathrm{P}<0.05$; Fig. 5).

ZO-1 expression. The TJ is also involved in intestinal mucosal barrier damage. In order to observe alterations in TJ proteins,

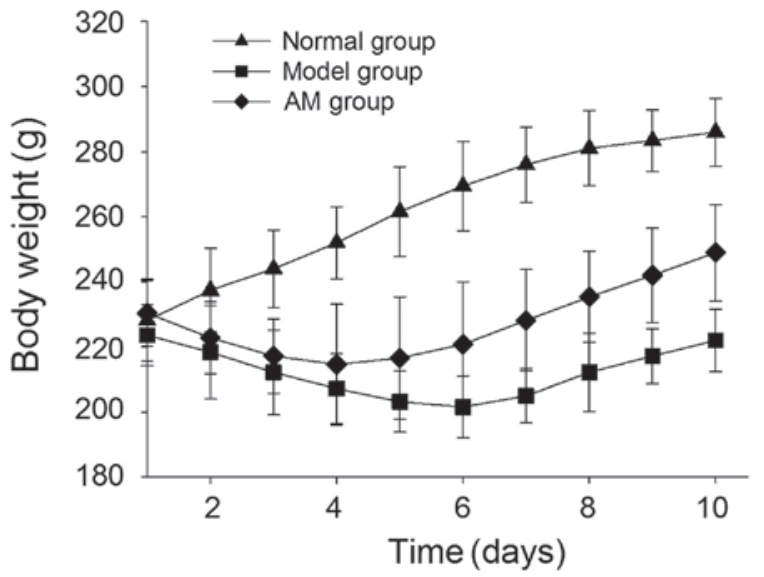

Figure 1. AM administration reduces weight loss in TNBS-induced colitis rats. A weight curve during TNBS-induced colitis for rats administered with saline and rats administered with AM was recorded. The results demonstrated an increasing weight of normal rats at the observation points. The body weight of rats significantly decreased in the model group with time, however, body weight significantly increased following AM treatment compared with the model rats. AM, adrenomedullin; TNBS, 2,4,6-trinitro-benzene-sulfonic acid.
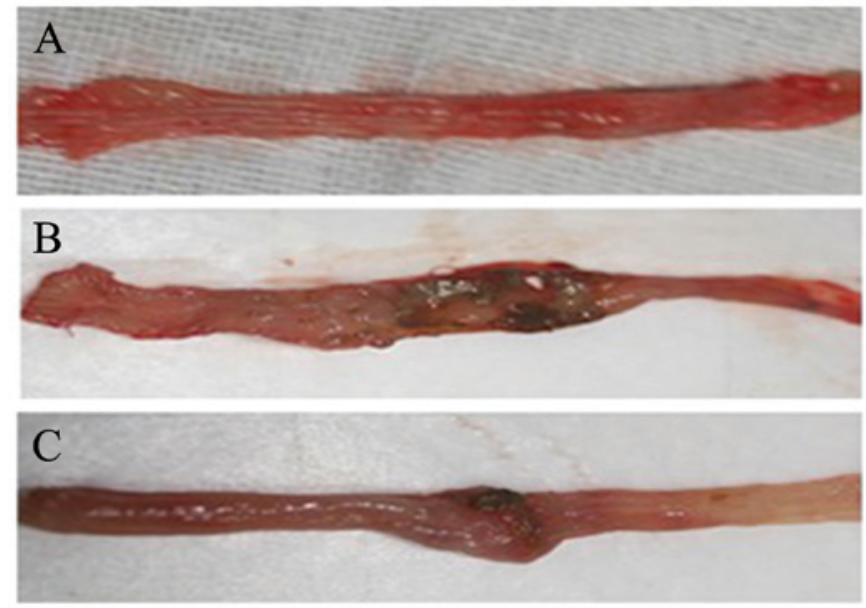

Figure 2. Visual inspection of colon tissues in rats. AM-administered rats were compared with the model group at the end of the experiment. (A) A normal morphology was observed in normal rats. (B) Bleeding and erosion of mucosa was observed in the model group. (C) Superficial ulcers of intestinal mucosa were significantly reduced following AM treatment. AM, adrenomedullin; TNBS, 2,4,6-trinitro-benzene-sulfonic acid.

the present study used western blot analysis to detect the expression level of ZO-1. Western blot analysis demonstrated a decreased expression of $\mathrm{ZO}-1$ in model rats, however, the expression of ZO-1 was decreased in the AM-treated group (Fig. 6).

TEM of TJs. Microvilli damage and TJ destruction are major contributors to epithelial dysfunction. In our experiments, TEM demonstrated the integrity of epithelial TJs and neat arrangement of microvilli in the normal group (Fig. 7A), however, a widened or fractured TJ, sparse microvilli and expansion of endoplasmic reticulum was observed in the model group (Fig. 7B). These were improved significantly following AM treatment (Fig. 7C). 


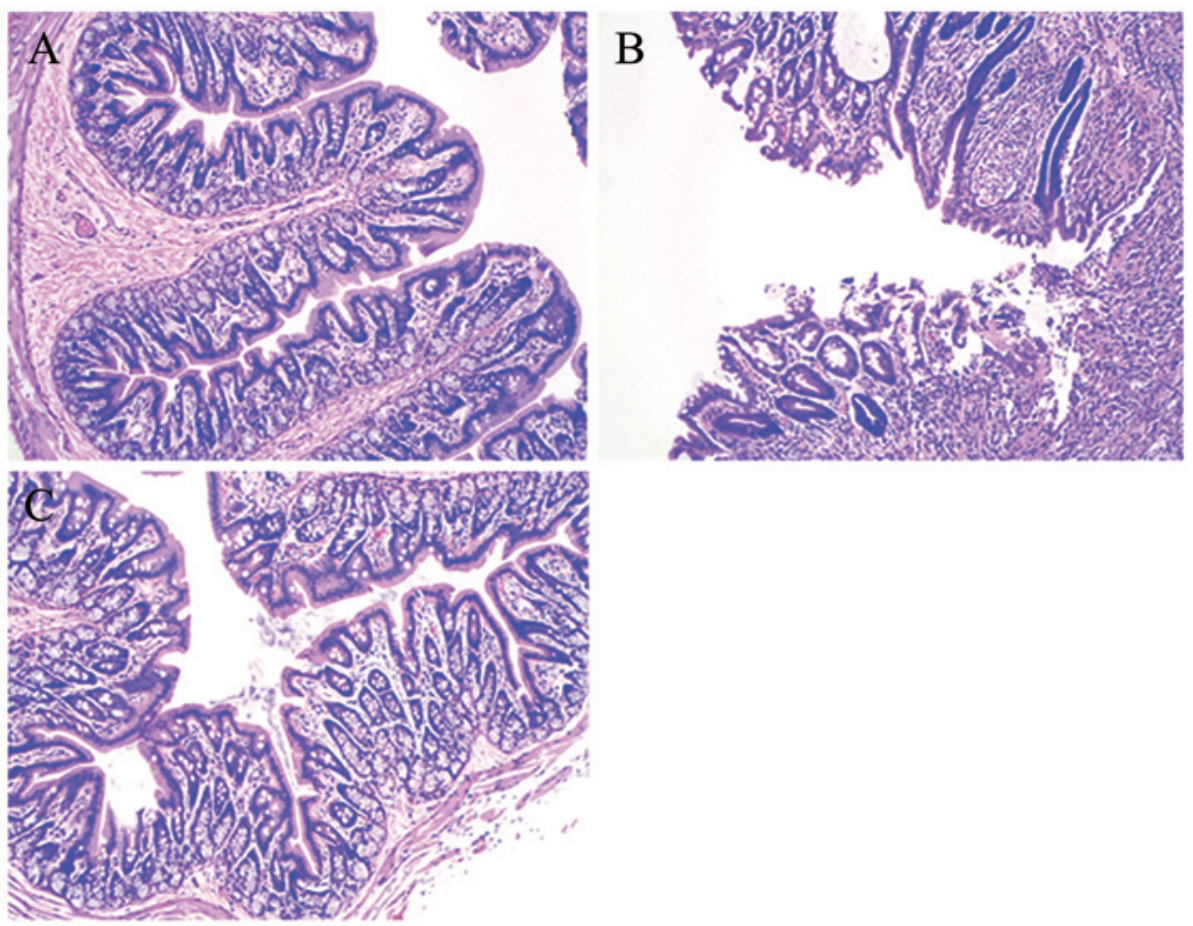

Figure 3. Effect of AM on histological alterations in colon tissue. Images show the staining of rat colon tissue sections with hematoxylin and eosin. (A) Clear intestinal wall structure and regularly arranged microvilli in the normal group (magnification, x100). (B) Model rats indicated severe mucosal defects and infiltration of the lamina propria by inflammatory cells (magnification, x100). (C) AM-treated rats demonstrated less mucosal defects and edema (magnification, x100). AM, adrenomedullin.

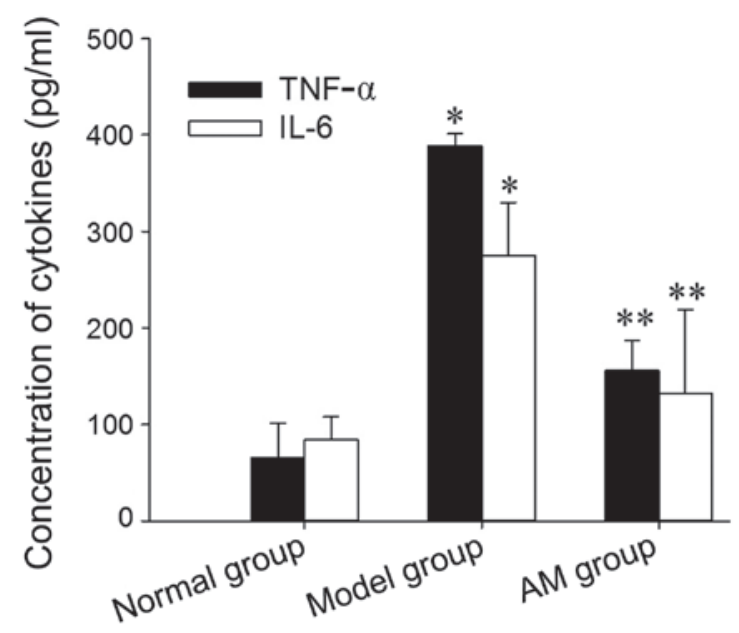

Figure 4. TNF- $\alpha$ and IL-6 assayed by ELISA using detection kits. ELISA was performed as described in the Materials and methods. Data are expressed as the mean \pm standard error of the mean. The expression of TNF- $\alpha$ and IL-6 in the serum of model rats was significantly higher than in normal rats $(\mathrm{P}<0.05)$. A lower expression of TNF- $\alpha$ and IL-6 was observed following AM treatment compared with the model group $\left({ }^{* *} \mathrm{P}<0.05\right)$. TNF- $\alpha$, tumor necrosis factor- $\alpha$; IL-6, interleukin-6; AM, adrenomedullin.

\section{Discussion}

IBD, including UC and Crohn's disease affects a large number of individuals. However, the specific mechanism involved remains to be elucidated. One important factor is intestinal mucosal barrier damage, which is often referred to as increased intestinal permeability $(1,2)$. There are different degrees of intestinal mucosal barrier dysfunction in a variety of IBDs and bacterial

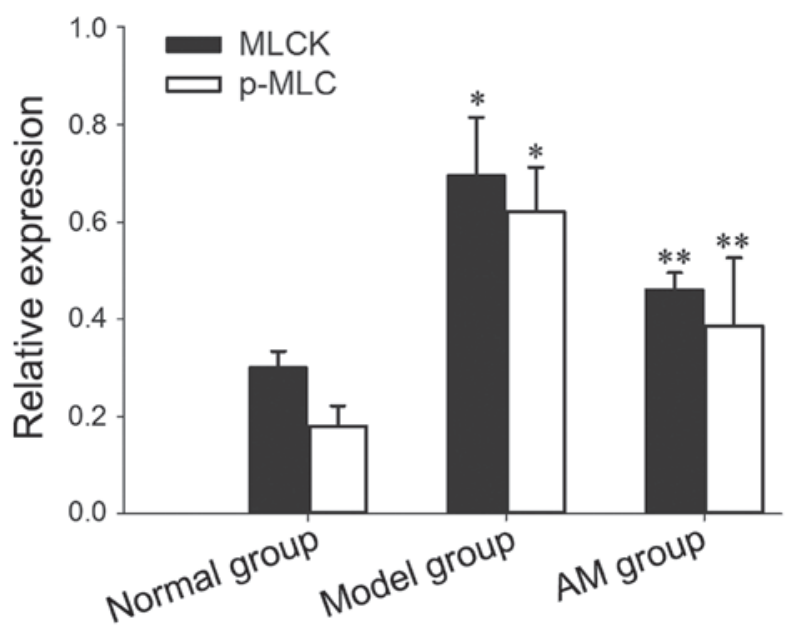

Figure 5. Effects of AM on mucosa MLCK and p-MLC protein expression levels in rat colon tissue. Proteins were collected and analyzed as described in the Materials and methods. Data are expressed as the mean \pm standard error of the mean. MLCK and p-MLC expression significantly increased compared with the normal rats $(" \mathrm{P}<0.05)$. Following AM administration, MLCK and p-MLC expression significantly decreased compared with untreated colitis rats $\left({ }^{* *} \mathrm{P}<0.05\right)$. AM, adrenomedullin; MLCK, myosin light chain kinase; p-MLC, phosphorylated myosin light chain.

dysentery. The integrity of the intestinal epithelial barrier has an important function to prevent electrolyte loss and toxin penetration (23). Inflammatory cytokines can alter the structure of intestinal epithelial TJs, resulting in impaired epithelial barrier function $(24,25)$. During the pathogenesis of IBD, TNF- $\alpha$ has been widely recognized as one of the key proinflammatory cytokines, which can increase paracellular permeability, damage 


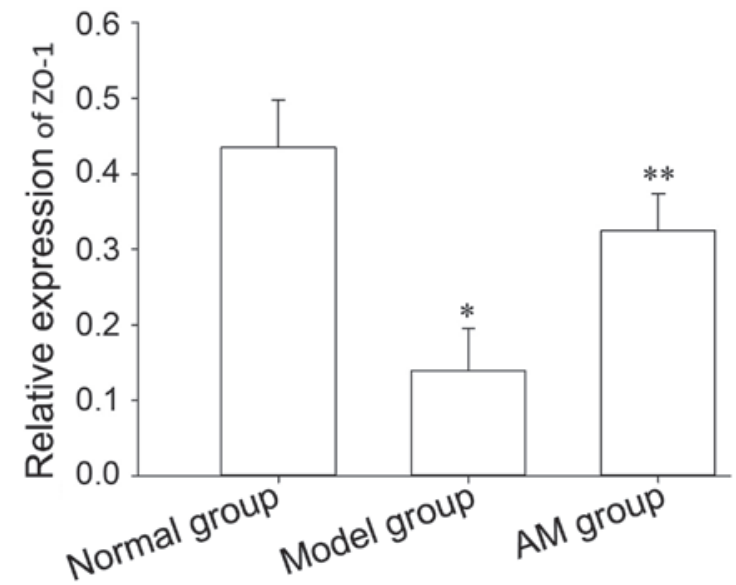

Figure 6. ZO-1 protein expression of rat colon samples. AM was administered via a rubber infusion tube of $1.0 \mu \mathrm{g}$ diluted in $1.0 \mathrm{ml}$ of saline once a day. Proteins were collected and analyzed as described in Materials and methods. Compared with the normal group, the expression of ZO-1 was decreased in the model group $\left({ }^{*} \mathrm{P}<0.05\right)$. Treatment with $\mathrm{AM}$ upregulated the expression of ZO-1 ( $\left.{ }^{* *} \mathrm{P}<0.05\right)$. ZO-1, zonula occludens-1; AM, adrenomedullin.
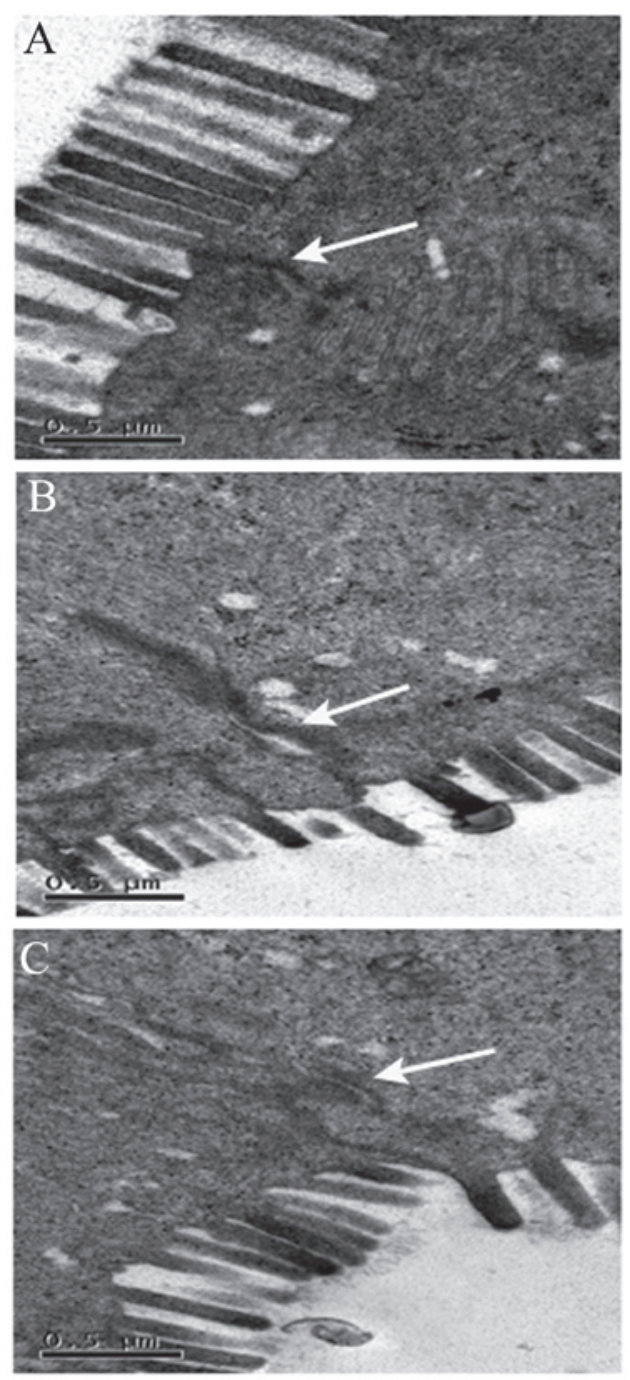

Figure 7. Intestinal epithelium ultrastructure was observed in the rats using transmission electron microscopy. (A) Normal microvilli and ultrastructure (scale bar $=0.5 \mu \mathrm{m}$ ). (B) Widened TJs and significant microvilli damage were observed in 2,4,6-trinitro-benzene-sulfonic acid-induced rats (scale bar $=0.5 \mu \mathrm{m}$ ). (C) TJs and microvilli clearly improved in adrenomedullin-administered rats (scale bar $=0.5 \mu \mathrm{m}$ ). TJs, tight junctions. the mucosal barrier and mediate intestinal inflammation, while TNF- $\alpha$ antagonist therapy can effectively alleviate intestinal inflammation and repair the intestinal mucosa tissues (26). TNF- $\alpha$ may be directly involved in intestinal barrier function damage. Previous studies have demonstrated that the dysfunction of intestinal epithelial cells induced by $\mathrm{TNF}-\alpha$ requires the involvement of MLCK and p-MLC, while inhibiting the synthesis of MLCK can significantly reduce the permeability of the epithelial barrier $(9,27,28)$, disclosing a key role of the MLCK-p-MLC pathway in intestinal epithelial barrier dysfunction induced by TNF- $\alpha$. In rat colitis induced by TNBS, an increased level of TNF- $\alpha$ was detected in the serum, at the same time, the protein expression levels of MLCK and p-MLC were also upregulated, however, this was prevented by the administration of AM, indicating its ability to inhibit activation of the MLCK-p-MLC pathway by TNF- $\alpha$.

In addition to TNF- $\alpha$, whether other cytokines are involved in the destruction of the gut barrier, remains to be elucidated. In the present study, serum IL-6 expression correlated with the increase of intestinal epithelial permeability in UC rats induced by TNBS. This result is also in agreement with a previous study demonstrating that IL-6 has a detrimental role in DSS-induced colitis (29). Notably, IL-6 may also be involved in the destruction of intestinal barrier function, demonstrating that AM may antagonize activation of the MLCK-p-MLC pathway by inhibiting expression of pro-inflammatory cytokines, including TNF- $\alpha$ and IL-6.

The intestinal mucosal barrier is important in maintaining intestinal function $(30,31)$. Previous studies have demonstrated that TNF- $\alpha$ can induce intestinal barrier dysfunction and the mechanism may be associated with the upregulation of MLCK expression (32). Turner et al $(1,33)$ demonstrated that TNF- $\alpha$-induced barrier dysfunction requires myosin II activation by MLCK-dependent MLC phosphorylation. Therefore, TNF- $\alpha$ can induce MLC phosphorylation and p-MLC has a crucial role in TNF- $\alpha$-induced barrier disruption. MLCK is a protein that catalyzes the phosphorylation of MLC, and the expression of MLCK was consistent with p-MLC. Our data revealed that MLCK and $\mathrm{p}-\mathrm{MLC}$ expression significantly increased compared with normal rats. Notably, ZO-1 protein expression clearly decreased and intestinal epithelial cell permeability increased at the same time. The MLCK-p-MLC pathway is not only essential for mucosal damage, but also causes epithelial barrier damage possibly through controlling the permeability of TJs.

$\mathrm{AM}$ is a type of endogenous vasoactive peptide, which is upregulated during inflammation in numerous areas $(14,34)$. In the treatment of IBD, AM has been confirmed to have a beneficial effect on the intestine by its anti-inflammatory and antibacterial properties and by suppressing inflammatory cytokines (17-19). In an intestinal ischemia-reperfusion injury rat model, AM was demonstrated to have a protective effect on intestinal mucosal structure and function (35). However, the effect of AM on MLCK and p-MLC of UC rats has not been reported. In our experimental colitis model induced by TNBS, the expression of TNF- $\alpha$, IL-6, MLCK and p-MLC significantly increased compared with normal rats. AM can decrease the level of TNF- $\alpha$ and IL-6, downregulate the expression of MLCK, inhibit the phosphorylation of MLC and improve the epithelial TJ so as to reduce intestinal mucosal permeability, indicating that AM 
possibly improves the permeability of the intestinal mucosa by inhibiting the expression of pro-inflammatory cytokines and reducing MLCK and p-MLC levels. Due to the complexity of the signaling pathway, only the MLCK-P-MLC pathway, which is closely associated with colonic mucosa permeability, was examined in the present study. Whether AM can mediate intestinal barrier dysfunction via other molecules, will be further investigated in subsequent experiments.

In conclusion, the current study demonstrated that AM treatment can improve intestinal mucosa permeability by downregulating the expression of proinflammatory cytokines and inhibiting activation of the MLCK-p-MLC pathway so as to ameliorate intestinal barrier function. The suppressive effects of AM on p-MLC may represent a mechanism responsible for the beneficial effects of AM. These findings further explain the pathogenesis of UC and may provide a new therapeutic strategy for the treatment of UC.

\section{Acknowledgements}

This study was supported by the Health and Family Planning Commission of Hubei Province (grant no. 2013Z-Y06).

\section{References}

1. Turner JR: Intestinal mucosal barrier function in health and disease. Nat Rev Immunol 9: 799-809, 2009.

2. Marchiando AM, Graham WV and Turner JR: Epithelial barriers in homeostasis and disease. Annu Rev Pathol 5: 119-144, 2010.

3. Munkholm P, Langholz E, Hollander D, Thornberg K, Orholm M, Katz KD and Binder V: Intestinal permeability in patients with Crohn's disease and ulcerative colitis and their first degree relatives. Gut 35: 68-72, 1994.

4. Ashizuka S, Inagaki-Ohara K, Kuwasako K, Kato J, Inatsu H and Kitamura K: Adrenomedullin treatment reduces intestinal inflammation and maintains epithelial barrier function in mice administered dextran sulphate sodium. Microbiol Immunol 53 573-581, 2009.

5. Gardiner KR, Anderson NH, Rowlands BJ and Barbul A: Colitis and colonic mucosal barrier dysfunction. Gut 37: 530-535, 1995.

6. Clayburgh DR, Shen L and Turner JR: A porous defense: The leaky epithelial barrier in intestinal disease. Lab Invest 84: 282-291, 2004

7. Gibson PR: Increased gut permeability in Crohn's disease: Is TNF the link? Gut 53: 1724-1725, 2004.

8. Nusrat A, Turner JR and Madara JL: Molecular physiology and pathophysiology of tight junctions. IV. Regulation of tight junctions by extracellular stimuli: Nutrients, cytokines, and immune cells. Am J Physiol Gastrointest Liver Physiol 279: G851-G857, 2000.

9. Ye D, Ma I and Ma TY: Molecular mechanism of tumor necrosis factor-alpha modulation of intestinal epithelial tight junction barrier Am J Physiol Gastrointest Liver Physiol 290: G496-G504, 2006.

10. Gu L, Li N, Gong J, Li Q, Zhu W and Li J: Berberine ameliorates intestinal epithelial tight-junction damage and down-regulates myosin light chain kinase pathways in a mouse model of endotoxinemia. J Infect Dis 203: 1602-1612, 2011.

11. Costantini TW, Loomis WH, Putnam JG, et al: Pentoxifylline modulates intestinal tight junction signaling after burn injury: Effects on myosin light chain kinase. J Trauma 66: 17-24; discussion 24-25, 2009.

12. Shen L: Tight junctions on the move: Molecular mechanisms for epithelial barrier regulation. Ann NY Acad Sci 1258: 9-18, 2012.

13. Liu X, Xu J, Mei Q, Han L and Huang J: Myosin light chain kinase inhibitor inhibits dextran sulfate sodium-induced colitis in mice. Dig Dis Sci 58: 107-114, 2013.

14. Elsasser TH and Kahl S: Adrenomedullin has multiple roles in disease stress: Development and remission of the inflammatory response. Microsc Res Tech 57: 120-129, 2002.

15. Sakata J, Asada Y, Shimokubo T, Kitani M, Inatsu H, Kitamura K, Kangawa K, Matsuo H, Sumiyoshi A and Eto T: Adrenomedullin in the gastrointestinal tract. Distribution and gene expression in rat and augmented gastric adrenomedullin after fasting. J Gastroenterol 33: $828-834,1998$
16. Ashizuka S, Ishikawa N, Kato J, Yamaga J, Inatsu H, Eto T and Kitamura K: Effect of adrenomedullin administration on acetic acid-induced colitis in rats. Peptides 26: 2610-2615, 2005.

17. Kataoka Y, Miyazaki S, Yasuda S, Nagaya N, Noguchi T, Yamada N, Morii I, Kawamura A, Doi K, Miyatake K, et al: The first clinical pilot study of intravenous adrenomedullin administration in patients with acute myocardial infarction. J Cardiovasc Pharmacol 56: 413-419, 2010.

18. Gonzalez-Rey E, Fernandez-Martin A, Chorny A and Delgado M: Therapeutic effect of urocortin and adrenomedullin in a murine model of Crohn's disease. Gut 55: 824-832, 2006.

19. Talero E, Alvarez de Sotomayor M, Sánchez-Fidalgo S and Motilva $\mathrm{V}$ : Vascular contribution of adrenomedullin to microcirculatory improvement in experimental colitis. Eur J Pharmacol 670: 601-607, 2011.

20. Hayashi Y,Narumi K, Tsuji S, Tsubokawa T,Nakaya MA, Wakayama T, Zuka M, Ohshima T, Yamagishi M and Okada T: Impact of adrenomedullin on dextran sulfate sodium-induced inflammatory colitis in mice: Insights from in vitro and in vivo experimental studies. Int J Colorectal Dis 26: 1453-1462, 2011.

21. Carrizo GJ, Wu R, Cui X, Dwivedi AJ, Simms HH and Wang P: Adrenomedullin and adrenomedullin-binding protein-1 downregulate inflammatory cytokines and attenuate tissue injury after gut ischemia-reperfusion. Surgery 141: 245-253, 2007.

22. Zuo D, Liu X, Shou Z, Fan H, Tang Q, Duan X, Cao D, Zou Z and Zhang L: Study on the interactions between transplanted bone marrow-derived mesenchymal stem cells and regulatory $\mathrm{T}$ cells for the treatment of experimental colitis. Int J Mol Med 32: 1337-1344, 2013.

23. Li X, Wang Q, Xu H, Tao L, Lu J, Cai L and Wang C: Somatostatin regulates tight junction proteins expression in colitis mice. Int J Clin Exp Pathol 7: 2153-2162, 2014.

24. Greig E and Sandle GI: Diarrhea in ulcerative colitis. The role of altered colonic sodium transport. Ann NY Acad Sci 915: 327-332, 2000.

25. Schmitz H, Barmeyer C, Fromm M, Runkel N, Foss HD, Bentzel CJ, Riecken EO and Schulzke JD: Altered tight junction structure contributes to the impaired epithelial barrier function in ulcerative colitis. Gastroenterology 116: 301-309, 1999.

26. Fischer A, Gluth M, Pape UF, Wiedenmann B, Theuring F and Baumgart DC: Adalimumab prevents barrier dysfunction and antagonizes distinct effects of TNF- $\alpha$ on tight junction proteins and signaling pathways in intestinal epithelial cells. Am J Physiol Gastrointest Liver Physiol 304: G970-G979, 2013.

27. Ma TY, Boivin MA, Ye D, Pedram A and Said HM: Mechanism of TNF-\{alpha\} modulation of Caco-2 intestinal epithelial tight junction barrier: Role of myosin light-chain kinase protein expression. Am J Physiol Gastrointest Liver Physiol 288: G422-G430, 2005.

28. Marchiando AM, Shen L, Graham WV, Weber CR, Schwarz BT, Austin JR 2nd, Raleigh DR, Guan Y, Watson AJ, Montrose MH, et al: Caveolin-1-dependent occludin endocytosis is required for TNF-induced tight junction regulation in vivo. J Cell Biol 189: $111-126,2010$

29. Sommer J, Engelowski E, Baran P, Garbers C, Floss DM and Scheller J: Interleukin-6, but not the interleukin-6 receptor plays a role in recovery from dextran sodium sulfate-induced colitis. Int J Mol Med 34: 651-660, 2014.

30. Irvine EJ and Marshall JK: Increased intestinal permeability precedes the onset of Crohn's disease in a subject with familial risk. Gastroenterology 119: 1740-1744, 2000.

31. Wyatt J, Vogelsang H, Hübl W, Waldhöer T and Lochs H: Intestinal permeability and the prediction of relapse in Crohn's disease. Lancet 341: 1437-1439, 1993.

32. Wang F, Graham WV, Wang Y, Witkowski ED, Schwarz BT and Turner JR: Interferon-gamma and tumor necrosis factor-alpha synergize to induce intestinal epithelial barrier dysfunction by up-regulating myosin light chain kinase expression. Am J Pathol 166: 409-419, 2005.

33. Taylor CT, Dzus AL and Colgan SP: Autocrine regulation of epithelial permeability by hypoxia: Role for polarized release of tumor necrosis factor alpha. Gastroenterology 114: 657-668, 1998.

34. Ueda S, Nishio K, Minamino N, Kubo A, Akai Y, Kangawa K, Matsuo H, Fujimura Y, Yoshioka A, Masui K, et al: Increased plasma levels of adrenomedullin in patients with systemic inflammatory response syndrome. Am J Respir Crit Care Med 160: 132-136, 1999.

35. Higuchi S, Wu R, Zhou M, Marini CP, Ravikumar TS and Wang P: Gut hyperpermeability after ischemia and reperfusion: Attenuation with adrenomedullin and its binding protein treatment. Int J Clin Exp Pathol 1: 409-418, 2008. 